\title{
La política exterior de los Estados Unidos en el último tercio del siglo XIX: una revisión historiográfica
}

\author{
Andrés SÁNChez PADilla \\ Universidad Complutense de Madrid \\ andsanch@ucm.es
}

Recepción: 14 de octubre de 2014 / Revisión: 25 de febrero de 2015

Aceptación: 30 de marzo de 2015 / Publicación: Diciembre de 2016

\section{RESUMEN}

Este artículo explora los últimos debates dentro de la historiografía norteamericana sobre la política exterior de los Estados Unidos en el último tercio del siglo XIX y los pone en relación con la historiografía española. Primero se repasan las aportaciones de las principales escuelas historiográficas norteamericanas, desde el realismo a los estudios culturales. Después se argumenta que la historiografía española no ha prestado la atención suficiente a lo que se ha escrito sobre la cuestión en los Estados Unidos. El artículo termina haciendo algunas sugerencias para mejorar el estado del debate académico a ambos lados del Atlántico.

Palabras clave: Historiografía, Estados Unidos, España, relaciones internacionales, política exterior, siglo XIX.

\section{United States Foreign Policy in the Last Third of the $19^{\text {th }}$ Century: a Historiographical Review}

\begin{abstract}
This article examines the latest debates in North-American historiography on the foreign policy of the United States in the last third of the 19th century, relating them to Spanish historiography. To begin with, the contributions of the principal historiographical schools, from Realism to Cultural Studies, are first reviewed. Subsequently, the case is made that Spanish historiography has not paid sufficient attention to what has been written about this in the United States. The article ends by providing some suggestions for the improvement of the state of the academic debate on either side of the Atlantic.
\end{abstract}

Keywords: Historiography, United States, Spain, International Relations, Foreign Policy, $19^{\text {th }}$ century.

SuMARIO: 1. Introducción. 2. El debate historiográfico en los Estados Unidos. 3. Los Estados Unidos en la historiografía española. 4. Conclusiones. 5. Referencias bibliográficas. 


\section{INTRODUCCIÓN}

El estudio de la política exterior de los Estados Unidos entre el final de la Guerra Civil norteamericana y el estallido de la Guerra de 1898 no ha despertado todavía demasiado interés en la historiografía española. Hasta ahora, los historiadores españoles se han mostrado más atraídos por la política exterior de Washington en el siglo $\mathrm{XX}$, un periodo en el que la influencia internacional de los Estados Unidos ha sido mucho más perceptible. En el siglo XIX, en cambio, la diplomacia norteamericana se concentró casi exclusivamente en problemas regionales que no afectaron al equilibrio del sistema internacional.

Sin embargo, no por eso la diplomacia norteamericana de las últimas décadas del Ochocientos merece arrojarse al olvido. Entre otras razones, porque los dilemas que han acosado a la política exterior estadounidense en el siglo XX adquirieron forma precisamente en ese periodo. De acuerdo con Walter LaFeber, "Modern American diplomatic history began in the 1850's and 1860's"'. Hasta 1865, la diplomacia de los Estados Unidos había estado condicionada por el conflicto doméstico sobre la esclavitud, y la propia supervivencia del experimento democrático norteamericano se había puesto en duda. Pero después de Appomattox los crecientes intereses estratégicos, económicos e ideológicos de los Estados Unidos empezaron a definir su política exterior ${ }^{2}$. De hecho, muchas potencias europeas dieron por sentado que después de su victoria sobre la Confederación, la Unión empezaría a participar activamente en la política europea. No fue así.

Durante las décadas que siguieron al final de la Guerra Civil, Washington siguió absteniéndose, salvo contadas excepciones, de los problemas europeos. Las breves intervenciones de los Estados Unidos en el continente tuvieron que ver con problemas coloniales, como la Conferencia de Madrid sobre Marruecos o la Conferencia de Berlín sobre el Congo.

En general, los gobiernos norteamericanos prefirieron concentrarse en sus áreas de influencia tradicionales: el Caribe y el Pacífico. Sin embargo, la política de Washington en esas áreas siguió sufriendo una falta de definición equiparable a la de las décadas previas a la Guerra Civil, aunque por diferentes motivos: las administraciones republicanas buscaron acuerdos comerciales y bases navales, habitualmente sin el apoyo del Congreso (con la excepción de Hawai'i); mientras que las administraciones demócratas trataron de derogar la mayoría de esos acuerdos. Ni siquiera en uno de los problemas que más ocupó la atención de la diplomacia norteamericana en esas décadas, la construcción de un canal interoceánico en el Istmo de Panamá, llegó a existir acuerdo entre demócratas y republicanos sobre si el gobierno federal debía patrocinar el proyecto. Por último, salvo excepciones (como en el caso de Corea o Chile), cuando los gobiernos norteamericanos tropezaron con otras potencias, prefirieron evitar los conflictos armados.

\footnotetext{
1 LA FEBER, 1963, p. 1.

2 Appomattox es la ciudad donde se produjo la rendición del ejército confederado del Norte de Virginia ante las tropas de la Unión el 9 de abril de 1865. Este hecho simboliza convencionalmente el final de la Guerra Civil norteamericana, aunque las tropas confederadas que operaban en el frente occidental del conflicto siguieron combatiendo durante varias semanas más.
} 
Explicar la inacción exterior de los Estados Unidos en ese periodo ha sido uno de los objetos de interés de la historiografía norteamericana desde hace varias décadas. En este artículo se examinarán las principales contribuciones norteamericanas a ese debate y su escaso eco en las discusiones españolas al respecto, señalando los desafíos pendientes en ambas historiografías. Para ello, primero estudiaré qué han dicho las diferentes escuelas historiográficas norteamericanas sobre los principios, los objetivos y las acciones de la política exterior de Washington en esta época ${ }^{3}$. A continuación, examinaré la persistencia de un modelo explicativo obsoleto de la política exterior norteamericana dentro de la historiografía española. Finalmente, se harán algunas sugerencias para mejorar el estado del diálogo historiográfico a ambos lados del Atlántico.

\section{EL DEBATE HISTORIOGRÁFICO EN LOS ESTADOS UNIDOS}

A pesar de su creciente poder, entre 1865 y 1898 los ejecutivos norteamericanos rechazaron habitualmente los compromisos internacionales, incluso en sus áreas de influencia tradicionales. Esa paradoja ha desconcertado a los historiadores norteamericanos desde hace mucho tiempo. De hecho, las primeras escuelas historiográficas que realizaron un esfuerzo por analizar sistemáticamente la política exterior de los Estados Unidos prefirieron ignorar las décadas anteriores a $1898^{4}$.

La escuela realista norteamericana dedicó cierta atención a la Guerra de 1898, que interpretó como una ruptura temporal con la realpolitik que había guiado a la república desde su nacimiento. De acuerdo con los realistas, la política de Washington en 1898 no respondió a los intereses nacionales debido a que generó costes muy superiores (especialmente, la aceptación de unos compromisos internacionales para los cuales los Estados Unidos aún no estaban preparados) a los beneficios obtenidos por la victoria sobre España. Para explicar este brusco viraje, los realistas sostuvieron que los líderes norteamericanos habían sucumbido a los sentimientos humanitarios de la población, hábilmente explotados por la prensa sensacionalista. Realistas más modernos, como Norman A. Graebner o Arthur M. Schlesinger, incorporaron a sus explicaciones la revalorización de los factores económicos y estratégicos producida con posterioridad, pero continuaron insistiendo en la primacía de los factores emocionales y en la ausencia de realismo de la política exterior norteamericana entre 1898 y $1900^{5}$.

En realidad, el interés en la política exterior de Washington durante el último tercio del Ochocientos fue impulsado por la escuela revisionista -también llamada escuela

\footnotetext{
3 El objeto de este artículo, por tanto, no es hacer una revisión de toda la historiografía norteamericana, sino sólo de la historiografía dedicada a las relaciones exteriores de los Estados Unidos. Por idénticas razones, tampoco se han incluido en la revisión los trabajos de ciencia política que no tengan un contenido histórico. Para una revisión del resto de la historiografía norteamericana, véase HigHAM, 1989; Novick, 1988.

4 ZAKARIA, 1998, pp. 48-55. Este olvido es aún más persistente entre los politólogos. Por ejemplo, RESTAD, 2012, también ignora por completo las tres últimas décadas del siglo XIX en su revisión de los paradigmas historiográficos sobre la política exterior norteamericana.

5 Graebner, 1985, pp. 313-355; SCHLESINGER, 1986.
} 
de Wisconsin, de la Puerta Abierta o, simplemente, New Left-, iniciada por William Appleman Williams en los años cincuenta ${ }^{6}$. Williams rescató del olvido las viejas tesis de historiadores progresistas como Harold U. Faulkner, Charles A. Beard o Leland H. Jenks sobre los fundamentos económicos de la diplomacia estadounidense ${ }^{7}$. Pero, a diferencia de sus antecesores progresistas, los historiadores revisionistas no establecieron una correspondencia tan exacta entre intereses económicos y acción exterior, modernizando el estudio de la política exterior norteamericana mediante el desplazamiento del énfasis analítico al contexto doméstico (en especial, el conflicto social) en el que los líderes y los diplomáticos tomaban sus decisiones ${ }^{8}$.

Por encima de otros factores domésticos, los autores revisionistas sostuvieron que el conflicto social era el principal motor de la expansión norteamericana, pero no en un sentido clásico. Los revisionistas defendían la existencia de un amplio consenso social en los Estados Unidos que sólo periódicas crisis de superproducción ponían en peligro. En ese contexto, la solución que mejor mantenía el statu quo social era la búsqueda incesante de mercados exteriores que aliviasen el exceso productivo. Sobre esta base, los revisionistas hicieron una reevaluación de toda la política exterior norteamericana para defender que, a pesar de las apariencias, existía una profunda continuidad estructural -con escasas diferencias entre demócratas y republicanos- en la política exterior de los Estados Unidos desde Appomattox a Vietnam.

Walter LaFeber, discípulo de Williams, fue el responsable de extrapolar el modelo revisionista al último tercio del siglo XIX con un argumento innovador: las décadas que precedieron a 1898 fueron "años de preparación" en los que la economía norteamericana se desarrolló extraordinariamente hasta que alcanzó tales niveles de superproducción en los años noventa que dieron lugar a la primera "gran depresión" de su historia, entre 1893 y 1897. Como única salida de la crisis, todos los intereses económicos (tanto agrarios como industriales) exigieron la obtención inmediata de mercados exteriores para exportar sus mercancías, pacíficamente o -si era necesario- por la fuerza. Cuba era uno de esos mercados, pero no el único. Sin embargo, la intransigencia de la política colonial española habría generado, a partir de 1895, un conflicto innecesario en la Gran Antilla que ponía en peligro la recuperación económica en los Estados Unidos.

LaFeber y Williams no sostuvieron que la Guerra de 1898 estuviese causada directamente por motivos económicos, sino que fue una pieza más de la diplomacia económica norteamericana en el Caribe y en el Pacífico: la administración McKinley buscaba ante todo mercados exteriores, pero la incapacidad de España para resolver el conflicto cubano ponía en peligro esa política y la intervención habría sido la solución menos problemática9 9

La influencia de este modelo explicativo revitalizó el estudio de la política exterior norteamericana en el Ochocientos durante la Guerra Fría. El trabajo de Walter LaFeber, en especial, inspiró multitud de estudios valiosos que ampliaron el conocimiento

6 Williams, 1959.

7 "Like their intellectual forebears of the 1920s, the new left identifies economic considerations as the central motivating factor in United States foreign policy", FRY, 1979, p. 92.

8 Sobre la escuela revisionista, véase BuZZANCO, 1999; FrY, 1979.

9 LAFEBER, 1963. 
sobre la diplomacia económica de Washington, como la tesis de James B. Chapin sobre la política exterior de la administración Grant ${ }^{10}$, que subrayó la importancia diplomática del tratado de comercio de 1875 con Hawai'i; o el estudio sobre los aranceles de Tom E. Terrill, que demostró la estrecha relación entre las batallas arancelarias en Washington y la política exterior norteamericana entre 1874 y $1901^{11}$.

Una de las razones del éxito del modelo revisionista fue la politización del debate historiográfico durante la Guerra Fría ${ }^{12}$. Pero ese éxito también se debía, en palabras de Paul S. Holbo, a que la aparente sencillez de su modelo ocultaba la complejidad de los problemas a analizar:

This thesis has the compelling appeal of simplicity. But its authors misread American political history and fail to note emotional and ideological ingredients that affected foreign policy ${ }^{13}$.

Lo cierto es que, a pesar de su materialismo, los autores revisionistas no habían prestado mucha atención a la evidencia económica y sus tesis fueron duramente rebatidas por ello: se puso de manifiesto, por ejemplo, que la gran industria norteamericana sólo empezó a interesarse por los mercados exteriores después, y no antes, de $1900^{14}$; que se había exagerado el clima intelectual expansionista anterior a 1898 mediante análisis retrospectivos ${ }^{15}$; y que el personal diplomático no había defendido eficazmente los intereses económicos norteamericanos en la mayoría de las ocasiones ${ }^{16}$. Finalmente, se llamó la atención sobre las proposiciones contradictorias que ofrecía el modelo revisionista al intentar dar una explicación unitaria de toda la política exterior norteamericana:

Professor Williams and his disciples are not committed to any single hypothesis about either the character or the causes of American foreign policy. Their apparently bold statements are much qualified and modified. The United States has been continuously and consciously 'expansionist' -but the forms and the techniques of that expansion have varied so greatly that antithetical policies can be seen as 'tactics' for achieving it. The basic reason for this expansion has been economic, but this does not imply that the American 'political economy' has actually depended upon overseas activity and trade, that American policy has always been the servant of particular American economic interests, or that American policy-makers have always consciously assigned a higher priority to economic goals than to strategic, political or ideological concerns. With the instinct of a modern theologian, Professor Williams seeks to incorporate all empirical objections into his theoretical framework. The result is that, by the time all the qualifications have been made, it is difficult to conceive of any American policy, or any evidence about the reasons for its adoption, which could not be incorporated

\footnotetext{
10 Chapin, 1971.

11 Terrill, 1973.

12 En palabras de FrY, 1979, pp. 92-93, “All new left scholars at least implicitly criticize United States policy for its aggressive nature and selfish, exploitative, economic goals".

13 Holbo, 1970, p. 207.

14 BECKER, 1973.

15 FiELD, 1978.

16 Pletcher, 1984.
} 
into the Williams interpretation. The hypothesis has come perilously closed to being non-falsifiable ${ }^{17}$.

Por su parte, la historiografía económica interesada en el estudio de la inversión extranjera también evidenció que las multinacionales norteamericanas surgidas en el último tercio del Ochocientos recibieron un escaso apoyo de Washington. En un trabajo pionero, Mira Wilkins reconstruyó el desarrollo de las primeras firmas norteamericanas en el exterior y descubrió que las más exitosas fueron las que no pidieron ni recibieron ayuda diplomática ${ }^{18}$. Por su parte, Robert B. Davies estudió la historia de Singer Manufacturing Company -fabricante de máquinas de coser y empresa multinacional líder hasta 1914-, poniendo de manifiesto que la empresa neoyorquina prefirió evitar el contacto con la diplomacia norteamericana en la mayoría de los casos ${ }^{19}$.

Igualmente crítico con la escuela revisionista, Philip S. Foner representó una de las pocas aportaciones marxistas al debate sobre la política exterior de Washington en el Ochocientos. Al igual que los revisionistas, Foner identificaba la economía como la variable explicativa fundamental de la diplomacia norteamericana en la crisis de 1898. Sin embargo, a diferencia de ellos, Foner defendía que la política exterior norteamericana nunca fue el producto de ningún tipo de consenso social: las administraciones de los presidentes Cleveland y McKinley se limitaron a inclinarse en todo momento ante los intereses económicos norteamericanos y a ignorar las aspiraciones del "pueblo" de los Estados Unidos. Mediante una interpretación ideologizada de las fuentes norteamericanas y cubanas, Foner reprodujo la vieja tesis marxista de que el desarrollo del capitalismo conducía mecánicamente al imperialismo: "The Cuban policy of the United States culminating in the use of force against Spain had its roots in the rise of monopoly capitalism and its drive for markets" ${ }^{20}$. Foner, por tanto, se limitó a actualizar con nuevas fuentes el viejo modelo explicativo de la historiografía progresista. No obstante, a pesar de su escasa novedad, el impacto de su obra en España ha sido muy superior al de cualquier otro historiador norteamericano, como veremos más adelante.

Una de las consecuencias del "fracaso" revisionista fue la de desincentivar la aparición de modelos explicativos alternativos ${ }^{21}$. En su lugar, la mayoría de los autores ha preferido reducir la escala de sus análisis ${ }^{22}$. Y ninguno de los historiadores que ha planteado nuevas interpretaciones ha tenido mucho éxito. Ni la síntesis de Robert L. Beisner entre los enfoques revisionistas y los de sus críticos desde una perspectiva sociológica, ni el modelo de Charles R. Lilley y Michael H. Hunt integrando a actores estatales y no estatales en tres esferas independientes, aunque relacionadas, ha conse-

17 Thompson, 1973, p. 104.

18 WiLKINS, 1970.

19 DAVIES, 1976.

20 FONER, 1972, p. 310.

21 Un "fracaso", en todo caso, relativo. Tal y como dice Joseph A. Fry, las tesis revisionistas "nonetheless continue to set the contours of discussion and analysis", FRY, 1996, p. 303. Y no sólo entre los historiadores: politólogos como RESTAD, 2012, siguen reproduciendo los argumentos revisionistas. Los autores revisionistas siguen en activo, aunque han introducido pocas variaciones en su modelo explicativo: LAFEBER, 1993; CRAPOL, 2000b; COATES, 2014.

22 CRAPOL, 2000a. 
guido demasiados seguidores ${ }^{23}$. Lo más cerca que se ha estado de un nuevo modelo general es la definición que David M. Pletcher dio de estas décadas como un periodo de transición:

What, then, was the significance of the period between 1865 and 1898 ? I suggest that this was simply a period of education, experimentation, and preparation but not of fulfillment ${ }^{24}$.

Pletcher ha formado parte de un grupo heterogéneo de historiadores que no ha rechazado todas las proposiciones de la escuela revisionista, pero sí ha cuestionado sus conclusiones. Desde los años sesenta, ha dedicado diversos estudios a la diplomacia económica norteamericana en los que ha puesto de manifiesto las contradicciones y la acción errática de los Estados Unidos en el exterior ${ }^{25}$. Por su parte, Lewis L. Gould ha señalado la dificultad de demostrar que la búsqueda de mercados exteriores fuera la prioridad de la administración McKinley durante la crisis de $1898^{26}$. Más recientemente, John L. Offner ha apuntado al escenario doméstico como el factor fundamental para explicar las acciones de la administración McKinley en 1897 y 1898, pero en un sentido muy distinto a los revisionistas. Según Offner, el objetivo fundamental del presidente republicano habría sido, por encima de todo, asegurar la hegemonía electoral de su partido a largo plazo ${ }^{27}$.

A partir de los años ochenta, a la insatisfacción interna con el estado del análisis sobre la política exterior norteamericana en el último tercio del siglo XIX se sumaron las críticas de otras especialidades sobre el provincianismo y la obsolescencia metodológica de la historia diplomática. Desde los estudios europeos, por ejemplo, se criticó repetidamente la ausencia del contexto internacional en la mayoría de los trabajos sobre la diplomacia norteamericana ${ }^{28}$. La respuesta a las críticas internas y externas fue variada.

Algunos autores intentaron, por ejemplo, extrapolar la teoría de la dependencia a las acciones de la diplomacia norteamericana en América Latina, con resultados desiguales. Louis A. Pérez aplicó este enfoque interpretativo en varios trabajos dedicados al estudio de los intereses económicos norteamericanos en Cuba, tanto antes como después de 1898. Sin embargo, aunque Pérez identificó correctamente los dilemas estructurales de la economía cubana, su análisis fue mucho menos convincente cuando trató de conectar los problemas cubanos con la política exterior norteamericana ${ }^{29}$.

El intento de Thomas D. Schoonover de extrapolar las teorías sobre los sistemas mundiales al análisis de la política exterior norteamericana entre Appomattox y Sa-

23 Beisner, 1986; Lilley - Hunt, 1987.

24 Pletcher, 1981, p. 104.

25 Pletcher, 1962; 1978.

26 GOULD, 1982.

27 OFFNER, 1992.

28 MAIER, 1980; MARKs, 1987, p. 266: "In the eyes of the Europeanists, the continuing debate over continuity and change in American foreign policy [...], and over isolationism versus internationalism and/or imperialism, is perfectly reasonable but hardly sufficient. The question remains of the effect of American policies on other countries and the reasons therefor".

29 PÉrez, 1983a; 1983 b. 
rajevo tampoco produjo resultados demasiado estimulantes debido a que se limitó a reproducir el modelo revisionista, pero sin prestar la misma atención al contexto doméstico de la diplomacia norteamericana ${ }^{30}$.

En los últimos años, Fareed Zakaria y Robert Kagan también han empezado a renovar las teorías realistas después de varias décadas de estancamiento. Zakaria ha elaborado un complejo modelo de análisis llamado "state-centered realism", de acuerdo con el cual los gobiernos norteamericanos entre 1865 y 1908 definieron su política exterior en función de una lógica autónoma y no sólo al servicio de intereses más o menos espurios. Zakaria ha desarrollado una interpretación que se apoya parcialmente en las tesis revisionistas para intentar explicar la inacción exterior de los Estados Unidos en esa época. De acuerdo con él, la sumisión del ejecutivo al Congreso y la debilidad militar de la república hasta finales de la década de 1890 impidieron que los Estados Unidos emprendiesen una agenda expansiva. Sólo cuando administraciones como las de Benjamin Harrison, William McKinley y Theodore Roosevelt fortalecieron el poder ejecutivo, los Estados Unidos estuvieron en condiciones de empezar a actuar agresivamente en la esfera internacional ${ }^{31}$.

Por su parte, Kagan ha insertado los principios ideológicos en la acción exterior de las administraciones norteamericanas decimonónicas, entendiendo la ideología como una variable expansionista. Para Kagan, los Estados Unidos se comportaron en el exterior como una potencia "revolucionaria" desde sus orígenes debido a su dedicación a la extensión internacional de los ideales universalistas sobre los que se habían fundado. La interpretación del triunfo de la Unión en la Guerra Civil como una cruzada abolicionista sólo habría reforzado esos ideales. Con el aumento del poder de los Estados Unidos en las décadas siguientes, habrían aumentado no sólo los intereses norteamericanos en el exterior, sino también los deseos de empezar a modificar el sistema internacional de acuerdo con los principios norteamericanos. La primera manifestación de esta conjunción de poder, intereses e ideología habría sido la Guerra de $1898^{32}$.

Pero, sin duda, la mayor renovación en el estudio de la política exterior de Washington en el Ochocientos se ha producido dentro de los estudios culturales ${ }^{33}$. Desde los años setenta, varios historiadores norteamericanos insatisfechos con los análisis revisionistas y con el estatocentrismo de la especialidad empezaron a plantearse el estudio de la dimensión cultural de las relaciones exteriores de los Estados Unidos, reinterpretando su política internacional como el resultado de la interacción entre sistemas de valores diferentes ${ }^{34}$.

Frank Ninkovich fue uno de los primeros autores que aplicó un análisis cultural a la diplomacia decimonónica para defender la persistencia de una ideología reticente (que denominó, irónicamente, "Open Door ideology") a la política de poder y la ex-

30 SCHOONOVER, 1991.

31 ZAKARIA, 1998.

32 Kagan, 2006.

33 Por razones de espacio, no es posible realizar un análisis detallado de la influencia del giro cultural en el estudio de las relaciones exteriores de los Estados Unidos. En las páginas siguientes me limitaré a revisar las contribuciones más relevantes sobre la política exterior norteamericana en el último tercio del siglo XIX.

34 IRIYE, 1979. 
pansión exterior. En sus palabras, "the Open Door ideology was designed to encourage commercial and cultural expansion while avoiding political entanglements" 35 . Es decir, al contrario que para Kagan, para Ninkovich la ideología habría retrasado, y no impulsado, la expansión de los Estados Unidos antes de 1917. De hecho, de acuerdo con él, incluso después de la entrada en la Primera Guerra Mundial, los líderes norteamericanos habrían continuado dudando sobre la dirección de la política exterior de su país.

Casi al mismo tiempo, Emily S. Rosenberg afirmó la existencia, desde finales del siglo XIX en adelante, de una ideología llamada "liberal-developmentalism" que habría guiado la política exterior norteamericana, con cinco características:

(1) Belief that other nations could and should replicate America's own developmental experience; (2) faith in private free enterprise; (3) support for free or open access for trade and investment; (4) promotion of free flow of information and culture; and (5) growing acceptance of governmental activity to protect private enterprise and to stimulate and regulate American participation in international economic and cultural exchange ${ }^{36}$.

Por su parte, Michael H. Hunt también defendió un rol determinante para la ideología en la política exterior de los Estados Unidos en el siglo XIX, aunque con un contenido diferente. Hunt identificó una potente ideología nacionalista como el motor de la expansión estadounidense, con tres puntos clave: la búsqueda de la grandeza nacional, el racismo y el miedo a la revolución ${ }^{37}$.

Todas estas teorías se acercan al modelo corporativista -heredero del revisionismo-, aunque la estrecha cooperación entre el gobierno y el sector privado que defiende son difíciles de aplicar al Ochocientos. De hecho, hasta ahora el corporativismo ha evitado extrapolar su modelo al siglo XIX ${ }^{38}$.

A pesar de la renovación que suponen, el principal problema que plantean todos los análisis culturales sigue siendo su ambivalencia sobre el rol causal de la ideología en particular, y del factor cultural en general, en la política exterior ${ }^{39}$. De hecho, los partidarios extremos del giro cultural se niegan a plantear el debate en términos de causalidad. En palabras de Volker Depkat:

There is a tendency among cultural studies scholars inspired by postmodernist theories to stop their work with deconstructing dominant discourses, with defining the limits of discursive formations and exposing their legitimatory function. As a result, they question the very possibility of intentional behavior; however, they do not really provide an answer for the question of why is it that people act the way they act ${ }^{40}$.

\footnotetext{
35 Ninkovich, 1982. Ninkovich ha ampliado sus argumentos en NinKovich, 1998.

36 RosenberG, 1982, p. 7.

37 Hunt, 1987.

38 Sobre el corporatismo, cfr. MCCORMick, 1982.

39 Ninkovich, 1998; SCHWARTZ, 2007.

40 DEPKAT, 2003, pp. 185-186.
} 
Algunos trabajos se conforman con establecer una correlación entre discurso y política exterior, como en el caso del papel de las ideologías de género en el estallido de la Guerra de $1898^{41}$. Pero descubrir que el género está presente en todos los discursos no añade una herramienta analítica muy útil:

Gender then hardly has any analytical meaning when it comes to explaining foreign relations. To claim that all aspects of past realities are culturally constructed is just as mundane as the somewhat traditional article of faith that men live in groups and that their behavior is therefore socially determined ${ }^{42}$.

A menudo, los análisis culturales están peligrosamente cerca de caer en la tentación de confundir correlación con causalidad. En palabras de Frank Ninkovich,

Cultural studies see discourses as fields of contestation. [...] But this desire to highlight the openness and arbitrariness of culture coexists uneasily with a desire to attribute to it a powerful coercive force ${ }^{43}$.

El principal problema analítico de este "determinismo" cultural es la incapacidad para explicar los cambios en la política exterior. Irónicamente, el análisis cultural puede llevar en ocasiones a un nuevo reduccionismo interpretativo a pesar de que su origen se encuentra precisamente en la insatisfacción que había generado el determinismo de las teorías materialistas precedentes ${ }^{44}$ :

For one thing, to what extent can domestic culture and ideologies, gender and sexuality, race and identity explain how or why the foreign policy of a given state evolved? Moreover, does a specific foreign policy evolve "because" of these cultural narratives? $[\ldots]$

If one wishes to argue that public narratives constitute a significant part of the decision-making processes, we need to know more about exactly which narratives influence decision making and how they function. Unless scholars can retrace the central role of cultural narratives in concrete situations of policy making, cultural approaches to foreign policy remain what they are: interesting but irrelevant ${ }^{45}$.

No todos los análisis culturales han evitado el problema de la causalidad. Al estudiar la misión que los Estados Unidos enviaron a Corea en 1871 para establecer

\footnotetext{
41 Hoganson, 1998.

42 DePKAT, 2003, p. 181; BuZzanco, 1999, p. 586, llega a una conclusión similar desde una posición revisionista: "gender assumptions and choice of words are principally a good starting point for analysis rather than conclusive in their own right. Certainly, American policymakers, overwhelmingly male and products of privileged backgrounds in most cases, were predisposed to think, act, and talk in a certain way, and it adds to our understanding of foreign policies if we examine the cultures from which these men came and the way they looked at the world. It seems, however, that masculine ideologies or language, which are artificially constructed and reflect larger values, tend to be "instruments" of policy rather than agents".

43 Ninkovich, 2011.

44 Para Ninkovich, en cambio, el reduccionismo no permite avanzar en ninguna dirección: "If we must have an ideological explanation, it must do justice to the actual complexities of ideological experience", NinKOVICH, 1982, p. 206.

45 DEPKAT, 2003, p. 182.
} 
relaciones formales con el reino asiático, Gordon $\mathrm{H}$. Chang establece una relación causal directa entre los prejuicios sobre Corea de los diplomáticos y militares norteamericanos y el estallido del conflicto entre ambos países, demostrando la primacía de los factores culturales sobre los económicos ${ }^{46}$.

Pero en muchos otros casos los análisis culturales han carecido de cualquier interés por la política exterior. Sin embargo, no parece posible entender completamente el rol del factor cultural en las relaciones exteriores norteamericanas sin diseccionar, previa o simultáneamente, el entramado de la diplomacia. Por el contrario, parece más razonable no excluir ninguna dimensión -ni cultural, ni política- si se quiere avanzar intelectualmente: "Until culture and power are somehow integrated conceptually and practically, as they are in real life, there will remain two cultures in the history of foreign relations" ${ }^{47}$.

En el caso del siglo XIX, la ausencia entre 1865 y 1898 de una política exterior definida ha facilitado la sustitución del análisis de la diplomacia por los modelos "culturales" sobre las relaciones exteriores de los Estados Unidos. La principal consecuencia ha sido la revalorización de las teorías del imperialismo para explicar la política exterior norteamericana. La historia "imperial" ha defendido que los Estados Unidos han sido un imperio casi desde el principio de su existencia ${ }^{48}$. Si bien la caracterización de Estados Unidos como un imperio no es algo novedoso en la historiografía norteamericana (se puede remontar al menos hasta los años veinte ${ }^{49}$ ), su creciente utilización en las últimas décadas ha ido de la mano de una considerable ampliación conceptual del término en un sentido cultural. Aunque se ha pretendido despolitizar la utilización del imperio como categoría historiográfica, a menudo se ha hecho a costa de evitar definir qué se entiende como tal. Por eso ha existido un escaso interés en precisar los límites y la naturaleza de ese "imperio" norteamericano. De hecho, muchos autores han preferido utilizar el imperio como una metáfora para explicar las relaciones exteriores de los Estados Unidos tanto en el presente como en el pasado.

Sin embargo, para Frank Ninkovich esa clase de metáforas entorpece más de lo que ayuda a los historiadores. Entre otras cosas, porque no tiene en cuenta el peso del "anti-imperialism" $o$ en la historia de los Estados Unidos ${ }^{50}$. Se podría añadir que no sólo no ha existido ningún esfuerzo de comparación con las potencias imperiales europeas, sino que ni siquiera ha habido un intento de contextualizar las acciones de los Estados Unidos con las de otros países. Con la excepción de Gran Bretaña.

Los últimos trabajos que han aplicado el modelo explicativo "imperial" a la política exterior norteamericana del último tercio del siglo XIX tienen en común su interés por el mundo británico. Autores como Jay Sexton, Marc-William Palen o Andrew Priest ${ }^{51}$ han reproducido la misma equiparación entre expansión imperial, comercio

46 Chang, 2003.

47 NinKovich, 2011.

48 Crapol, 2000a.

49 FrY, 1979, pp. 77-98; Pletcher, 1990.

50 NinKovich, 2010.

51 Tanto Palen como Priest son historiadores británicos, pero se incluyen en calidad de especialistas en la política exterior de los Estados Unidos. 
internacional e influencia ideológica que defendieron los revisionistas ${ }^{52}$. Su aportación más novedosa es su defensa, basada exclusivamente en fuentes norteamericanas y británicas, de que la influencia del modelo imperial británico en la política exterior norteamericana fue muy superior a lo que se había dado por supuesto hasta ahora, bajo la premisa de que la política internacional del siglo XIX fue, ante todo, un mundo británico.

Sexton, por ejemplo, ha defendido la existencia de un proceso de emulación entre los Estados Unidos y Gran Bretaña en sus trabajos sobre las reclamaciones del Alabama y sobre la iniciativa multilateral de $1875^{53}$. Palen, por su parte, ha estado estudiando la diplomacia económica entre Gran Bretaña y los Estados Unidos y ha defendido que el Arancel McKinley tuvo un impacto decisivo en las colonias británicas de habla inglesa y que, por su parte, los lobbies librecambistas británicos habrían ejercido una influencia más considerable de lo que se había supuesto en la política comercial de los Estados Unidos. Asimismo, Palen ha desarrollado una nueva teoría para explicar la expansión norteamericana entre 1890 y 1913 que ha denominado "the imperialism of economic nationalism". Esta teoría responsabiliza exclusivamente a los republicanos por las políticas expansionistas inauguradas por el Arancel McKinley ${ }^{54}$. Finalmente, Priest ha reiterado la influencia británica en la política de los Estados Unidos en Cuba durante la Guerra de los Diez Años (1868-1878). Según él, durante ese conflicto Washington ensayó exitosamente en Cuba por primera vez el imperialismo informal que había aprendido de los británicos, aunque ese ensayo sólo habría dado completamente sus frutos una vez implantó su hegemonía en todo el Caribe a partir de $1898^{55}$.

\section{LOS ESTADOS UNIDOS EN LA HISTORIOGRAFÍA ESPAÑOLA}

La historiografía española ha dedicado una atención insuficiente a la política exterior de los Estados Unidos en el último tercio del siglo XIX. A pesar de la importancia de las relaciones con los Estados Unidos para entender el problema cubano, ni los historiadores internacionalistas ni los americanistas han tenido mucho interés en bucear en el ángulo norteamericano de esa cuestión.

Igualmente, no se ha establecido ningún diálogo con los historiadores estadounidenses. De hecho, ni siquiera se puede decir que haya existido un debate español sobre la política exterior de los Estados Unidos. Ciertamente, la historiografía norteamericana nunca ha prestado mucha atención a lo que se ha escrito desde España sobre su política exterior, pero a estas alturas todavía sigue siendo frecuente que los historiadores españoles hagan generalizaciones sobre la política exterior norteamericana sin tener un conocimiento aceptable de lo que se ha escrito en los Estados Unidos.

\footnotetext{
52 Algo que también criticó Thompson hace tiempo: Thompson, 1973, p. 104.

53 SEXTon, 2003; 2006. La iniciativa multilateral de noviembre de 1875 fue una maniobra de los Estados Unidos durante la Guerra de los Diez Años (1868-1878) para implicar a las grandes potencias europeas en la pacificación de Cuba. Fracasó rápidamente debido a que ninguna de ellas se sumó a la iniciativa.

54 Palen, 2010; 2013; 2015.

55 PRIEST, 2014.
} 
Este retraso resulta difícil de explicar. Podría ser un producto de la persistencia en la historiografía española de lo que Enric Ucelay-Da Cal ha denominado "the Hispanic Studies 'ghetto", un tipo de actitud profesional por el cual "Spanish historians within Spain assume a rigid attitude in the defense of national history, which tends to obviate any manner of connection with outside subjects" 56 .

Para Sylvia L. Hilton, el problema reside en que en España, "The great majority of historical studies are concerned with the United States only insofar as it has been directly linked to the historical development of Spain itself"57. Para Adrian Shubert, esta orientación "ensimismada" de la historiografía española también es consecuencia de la endogamia del sistema universitario:

With very few exceptions, people are hired for teaching jobs by the department in which they did their doctorate, and very often their first degree as well. In theory, all positions are open to qualified applicants; in reality 'outsiders' rarely get a look-in, and upcoming positions are recognized as being for 'specific' people. In such a system, merit in the usual sense becomes a secondary consideration. Even this degree of endogamy is further exacerbated by the fact that any departure from the usual track, such as spending time at an institution abroad, is more often seen as a liability than an $\operatorname{asset}^{58}$.

El elemento fundamental del modelo explicativo que usan los historiadores españoles para interpretar la política exterior norteamericana es la primacía que se otorga a la expansión de los Estados Unidos, sea económica o territorial. Esta primacía se debe a que la mayoría de los historiadores españoles que ha hecho referencia a la diplomacia norteamericana se ha apoyado casi exclusivamente en el trabajo de la historiografía cubana. El único historiador norteamericano citado con frecuencia es Philip S. Foner ${ }^{59}$. A diferencia de los demás historiadores norteamericanos, Foner ha disfrutado de la ventaja de estar traducido al castellano ${ }^{60}$.

Como resultado, la mayoría de los trabajos españoles ha perpetuado una imagen extremadamente simplificada de la política exterior norteamericana: uniforme, agresiva y en constante búsqueda de territorios o mercados de exportación. Por supuesto, este modelo explicativo tampoco presta ninguna atención al contexto doméstico en el que se movieron los líderes norteamericanos. Quizá nadie lo haya descrito mejor que Sylvia L. Hilton:

56 UCELAY-DA CAL, 1994, p. 12. Asimismo, añade que "Spanish historiography today (including Catalan historians, Basques, Galicians, etc) is almost universally hostile to outside work, especially in the English language and especially from the United States" (p. 13). A día de hoy, esta hostilidad es cada vez menor, pero sigue estando presente en muchos de los trabajos que hacen referencia a la política exterior de los Estados Unidos y el 98. Este fenómeno también podría ser consecuencia de la tendencia de la historiografía española sobre el 98 a escribir "judgemental works, which attribute responsibilities for the 'disaster"”, HiLton, 1994 a, p. 97.

57 Hilton, 1994b, p. 44. Más recientemente, HiLton, 2007, ha llamado la atención sobre el aumento en España de los cursos dedicados a la historia de los Estados Unidos desde 1975, aunque señalando que la mayoría de ellos es impartida por filólogos o latinoamericanistas. La escasa especialización de los docentes puede explicar la ausencia de resultados académicos apreciables hasta la fecha.

58 SHUBERT, 2004, pp. 361-362.

59 Companys, 1991; Robles, 1991; Roldán, 2000; Rubio, 2004; SAntamaría - Naranjo, 1999.

60 Dos veces: en Madrid (Foner, 1975) y en La Habana (Foner, 1978). 
There is still little in-depth knowledge of social, economic, and political conditions in the United States. There is, to be sure, a much greater familiarity with American historiography on the war of 1898, but even so, little sense of the complexity of American points of view. The United States and/or President McKinley are still often personified as the villain. References to "expansionism" make much of the attitudes and interventions of Henry Cabot Lodge and Theodore Roosevelt, with perhaps a reminder of Alfred Mahan's ideas thrown in for good measure, but little detailed explanation of how these influences connect with policy decisions. Likewise, it is commonplace to find references to the historical interest shown by different American governments and interest groups in the acquisition of Cuba throughout the nineteenth century, and such precedents are taken as sufficient explanation of the events of 1898.

Most authors mention "economic motives" (sometimes identified simply as "American business" or as sugar interests) -the need for foreign investment markets to place excess American capital and/or for Caribbean and Far Eastern markets as outlets for American agricultural and industrial surpluses. Yet again, they do not clearly show the connections between such interests and actual policy. Spanish historians, like their American colleagues, may portray McKinley as a weak politician or as a determined expansionist, but rarely do they back up these evaluations with any relevant information about American domestic and party politics, regional characteristics and tensions, the relationship between Congress (particularly the Senate) and the President, or examples of McKinley's handling of journalists, representatives of special interest groups, and public opinion in general ${ }^{61}$.

Desde que en los años veinte Jerónimo Bécker dedicó a los Estados Unidos varios capítulos de su voluminoso estudio sobre la política exterior española en el siglo XIX, el interés español en la diplomacia norteamericana del Ochocientos se ha concentrado casi exclusivamente en la Guerra de $1898^{62}$.

Los historiadores españoles que más espacio han dedicado a las relaciones hispano-norteamericanas han sido los especialistas en la historia de las relaciones internacionales. Javier Rubio es uno de los pocos historiadores internacionalistas que ha dedicado algunos trabajos a la política exterior de los Estados Unidos antes de 1898, aunque su interés se ha limitado a descontextualizar una serie de episodios aislados. En sus obras ha estudiado, entre otros asuntos, el Manifiesto de Ostende de 1854, las negociaciones para la emancipación de Cuba de 1869 y 1870, la crisis del Virginius de 1873 , la iniciativa multilateral de 1875 , las negociaciones comerciales de 1883 y 1884, las reclamaciones norteamericanas de los años noventa y la voladura del U.S.S. Maine en $1898^{63}$.

Pero en sus trabajos Rubio apenas ha tenido en cuenta los debates historiográficos norteamericanos más recientes. En su lugar, ha preferido construir su análisis sobre la base de trabajos mucho más antiguos, como los de French E. Chadwick o Allan Nevins. Así, de acuerdo con él, todas las administraciones norteamericanas persiguie-

\footnotetext{
61 Hilton, 1998, p. 15.

62 BÉCKER, 1924-1926.

63 Rubio, 1995; 2004.
} 
ron la misma política imperialista en Cuba entre 1853 y 1898. Buscando siempre la expansión territorial a toda costa, sólo habrían variado en los medios utilizados.

El ejemplo más representativo del nivel analítico de Rubio es el tratamiento que da a la explosión del U.S.S. Maine. Rubio llega al extremo de sugerir que la administración McKinley habría orquestado deliberadamente el accidente del buque con la intención de obtener el casus belli que necesitaba para declarar la guerra a España. Pero no se queda ahí. De acuerdo con su análisis, llega a la conclusión de que, de una u otra manera, los Estados Unidos fueron los responsables indirectos de la explosión de su propio acorazado y que, por tanto, debía haber sido su gobierno el que abonase a España los costes de la reparación de los daños que la explosión causó en el puerto de La Habana. Rubio concluye su análisis criticando acerbamente el escaso espacio que la historiografía norteamericana ha dedicado a este episodio, sugiriendo que la mayoría de los historiadores estadounidenses habría estado empeñada en una conspiración de silencio para ocultar la responsabilidad de su propio gobierno en el accidente ${ }^{64}$. Significativamente, todos los trabajos de Rubio han sido publicados por la editorial del Ministerio de Asuntos Exteriores.

El resto de los historiadores internacionalistas españoles ha preferido limitar su atención a la Guerra de 1898. Julián Companys, por ejemplo, sigue siendo la referencia con un análisis más polémico que académico de la misión diplomática de Steward L. Woodford en España (1897-1898), también publicado por el Ministerio de Asuntos Exteriores. Al igual que Rubio, Companys también entiende que la historiografía norteamericana moderna no ha aportado nada relevante a la cuestión y prefiere estudiar las relaciones hispano-norteamericanas a partir de una breve selección de las misivas que Woodford envió al Departamento de Estado y al presidente McKinley entre 1897 y 1898 . Sobre esa base, Companys, al igual que Rubio, caracteriza la política exterior norteamericana como una búsqueda incesante de territorios por todos los medios a su alcance ${ }^{65}$.

Cristóbal Robles también estudia la política exterior norteamericana en 1898, pero utilizando exclusivamente documentación española y vaticana. A diferencia de Companys, Robles evita el análisis polémico, pero a costa de cualquier estructura argumental. Se limita a volcar los testimonios de la época en un collage confuso, sin llegar a distinguir claramente entre sus puntos de vista y los de sus fuentes. No obstante, se cuida de reiterar repetidamente la agresividad de la política exterior norteamericana ${ }^{66}$.

Los trabajos más recientes no han mejorado visiblemente la situación. Elena Castelló, por ejemplo, reproduce todos los lugares comunes sobre la política exterior de

64 Rubio, 2004, pp. 1.167-1.209. Si Rubio conociese mejor la historiografía norteamericana, se habría dado cuenta de que la disminución del espacio dedicado al episodio del U.S.S. Maine no se debe a ningún tipo de conspiración, sino al creciente consenso sobre su escaso peso relativo en el estallido de la Guerra de 1898 , véase PÉREZ, 1989.

65 Companys, 1991. Hilton, 1994a, pp. 107 y 113, señala que los historiadores españoles suelen utilizar con frecuencia el término "yankees", con obvias connotaciones peyorativas, para referirse a los norteamericanos durante la Guerra de 1898. Companys representa un ejemplo extremo de esta tendencia, pues no sólo usa indiscriminadamente el término "yankees", sino que caracteriza a los norteamericanos como personas ingenuas, ignorantes, fáciles de engañar por parte de la prensa y con escasa memoria.

66 Robles, 1990; 1991. 
los Estados Unidos en el capítulo que dedica a la misión de Juan Valera en Washington (1884-1886) dentro de su biografía de escritor y diplomático ${ }^{67}$. Por su parte, Juan Pando, Agustín Sánchez Andrés y Juan Antonio Inarejos sólo mencionan ocasionalmente a los Estados Unidos en sus estudios sobre las conversaciones entre Juan Prim y Daniel E. Sickles, la política española en la cuenca del Caribe entre 1865 y 1898, y las intervenciones coloniales de la Unión Liberal, respectivamente ${ }^{68}$.

Mención aparte merece la voluminosa historiografía generada por el centenario de la Guerra de 1898. 1998 podía haber supuesto una buena ocasión para que la historiografía española empezase a tomar nota de las innovaciones que se habían producido al otro lado del Atlántico. No fue así ${ }^{69}$. En este sentido, el centenario sirvió, una vez más, para exhibir la predilección de muchos historiadores españoles por la reiteración de viejas interpretaciones durante la conmemoración de efemérides. El resultado fue una avalancha de publicaciones que no añadió mucho al conocimiento sobre la política exterior de los Estados Unidos antes del estallido del conflicto hispano-norteamericano ${ }^{70}$.

Una de las carencias más llamativas fue la escasa colaboración con historiadores norteamericanos. El pequeño esfuerzo emprendido se vio lastrado por la necesidad de traducir al castellano a los especialistas con los que se contactó, que se limitaron a reproducir en esta lengua trabajos previos en inglés ${ }^{71}$. John L. Offner fue el único especialista en la política exterior norteamericana que colaboró en el libro colectivo coordinado por Juan Pablo Fusi y Antonio Niño con una apretada síntesis de su trabajo sobre la Guerra de $1898^{72}$. En el resto de los casos, se prefirió contar con hispanistas o especialistas en la historia social y política de los Estados Unidos que no escribieron específicamente sobre la crisis de 1898: fue el caso de Bruce Laurie, Michael H. Frisch, Christopher Schmidt-Nowara y David Thelen en los números de la revista Studia Historica. Historia Contemporánea publicados en 1998 y $1999^{73}$. Asimismo, Schmidt-Nowara colaboró en el libro colectivo dirigido por Juan Pan-Montojo sobre la crisis de 1898. Característicamente, en un trabajo monográfico sobre 1898 la única colaboración norteamericana es obra de un hispanista que escribe un capítulo sobre el colonialismo español ${ }^{74}$.

Sólo alguno trabajos escaparon de este patrón, como los de Sylvia L. Hilton y Steve J.S. Ickringill sobre la disputa anglo-venezolana en la Guayana Británica y el

\footnotetext{
67 Castelló, 2009, pp. 541-712.

68 InAREJos, 2010; PANDO, 1996; SÁNCHEZ ANDRÉs, 1998; 2007.

69 Los repertorios bibliográficos publicados entonces son un buen ejemplo de la escasez del esfuerzo para ponerse al día con la historiografía norteamericana, $c f r$. VV.AA., 1998.

70 Dos balances exhaustivos en ElizALde, 2000; 2001.

71 Como ha señalado UCELAY-DA CAL, 1994, pp. 12-13, muchos historiadores españoles todavía sufren "“the fallacy of an imperial language' [...] Spanish historians consciously or unconsciously expect to read in Spanish abroad".

72 OFFNER, 1997.

73 Laurie, 1998; Frisch, 1998; Schmidt-Nowara, 1999; Thelen, 1999. El número de 1999 también incluye un artículo de un historiador británico: LANGHORNE, 1999.

74 SCHMIDT-NowARA, 1998.
} 
papel de la doctrina Monroe en la crisis de $1898^{75}$; o el estudio de M. ${ }^{a}$ Dolores Elizalde sobre la política norteamericana en el Pacífico en 1897-189876.

Fuera de las relaciones internacionales, la historiografía americanista tampoco ha prestado la atención suficiente a los Estados Unidos. Mientras que existe un conocimiento amplio y profundo de la historiografía cubana, no se ha realizado un esfuerzo recíproco con la historiografía norteamericana. Eso ha hecho que la perspectiva cubana coloree todos los análisis sobre los Estados Unidos, desequilibrio que lastra muchos trabajos. No resulta satisfactorio, por ejemplo, que se citen libros de texto sobre Estados Unidos como obras de referencia ${ }^{77}$, que se confunda el bill McKinley con el Arancel McKinley ${ }^{78}$, el Arancel McKinley con el Arancel Wilson-Gorman ${ }^{79}$, o que se afirme repetidamente que España accedió a todas las demandas estadounidenses en abril de $1898^{80}$. Estos errores fácticos no son banales, porque perpetúan la interpretación reduccionista de la política exterior de los Estados Unidos.

En este sentido, es significativo que el espacio que la principal revisión historiográfica sobre "el 98 en América" dedica a la política exterior de los Estados Unidos sea tan parco y esté tan poco al día ${ }^{81}$. A pesar de proclamar que "el criterio de selección [de su artículo] no es la nacionalidad, sino la actualidad y el estado de la cuestión", Antonio Santamaría y Consuelo Naranjo entienden que hasta 1996 las obras de referencia sobre la política norteamericana en Cuba fueron las escritas por autores cubanos (con la excepción de autores como Foner y Jenks); mientras que de la producción posterior a 1996 sólo son dignos de mención aquellos estadounidenses que han sido traducidos al español. Asimismo, de las revisiones historiográficas realizadas en los propios Estados Unidos, sólo mencionan la de Paterson ${ }^{82}$, un autor que ni siquiera es especialista en la época pero que, una vez más, cuenta con la ventaja de estar traducido al castellano. Con estos mimbres, no es de extrañar que ambos autores puedan afirmar que:

Nadie discute que su especialización [la de Cuba] en producir azúcar, cuya venta se concentró progresivamente en el mercado norteamericano desde mediados del siglo XIX, es clave para explicar la guerra de independencia y el protectorado impuesto en Cuba por los EE.UU., que tuvo como contrapartida un tratado comercial que favorecía dicha venta ${ }^{83}$.

75 HiLTON - ICKRINGILL, 1996; 1998.

76 ElizALDE, 1997. Elizalde ya había aportado mucha información sobre la política de los Estados Unidos en ese área en su monografía sobre la colonia española de las Islas Carolinas. ElizALDE, 1992.

77 Piqueras, 2003, p. 162.

78 GonZÁlez MarTíNEZ, 1998, p. 27.

79 Fradera, 1998, p. 198.

80 Sobre la repetición de esta acusación por parte de los historiadores españoles, HiLton, 1994a, pp. 112114. GouLD, 1985, pp. 184-185, explica elocuentemente por qué la suspensión de hostilidades que ofreció España no era equivalente al armisticio solicitado por los Estados Unidos.

81 Santamaría - Naranjo, 1999.

82 PATERson, 1996.

83 Santamaría - Naranjo, 1999, p. 217. 
El Arancel McKinley ejemplifica los problemas a los que lleva este reduccionismo. El llamado bill McKinley (con el que se suele confundir el arancel ${ }^{84}$ ), aprobado por la Cámara de Representantes en abril de 1890, era una legislación proteccionista que elevaba considerablemente los derechos de entrada del azúcar extranjero, incluido el de Cuba. No era una medida dirigida a conquistar mercados exteriores: ante todo, era una medida dirigida a proteger todavía más el mercado doméstico norteamericano de la competencia extranjera. En cambio, el Arancel McKinley aprobado por las dos cámaras del Congreso y sancionado por el presidente en octubre de ese año incluía algunas cláusulas adicionales que autorizaban al presidente a conceder franquicias sobre los derechos de importación a los productos extranjeros importados desde países que concediesen reducciones equivalentes a las mercancías norteamericanas. Esas cláusulas sólo habían sido introducidas en el Senado y obtenido la confianza de las cámaras gracias a una fuerte campaña de presión de la administración republicana del presidente Harrison. A pesar de ello, el Arancel McKinley nunca dejó ser una medida controvertida. La oposición demócrata tachó las cláusulas del arancel de inconstitucionales y denunció la legislación ante el Tribunal Supremo, que acabó fallando a favor de la administración en Field v. Clark (1892). No obstante, los demócratas utilizaron el arancel como arma arrojadiza en las elecciones legislativas de 1890 y en las elecciones presidenciales y legislativas de 1892. Después de sus victorias electorales en ambos comicios, acabaron sustituyendo el Arancel McKinley por una legislación menos proteccionista, el Arancel Wilson-Gorman, en 1894. El nuevo arancel derogó de un plumazo todos los acuerdos de reciprocidad firmados por la administración Harrison ${ }^{85}$.

Este complejo proceso tiene poco que ver con la visión monolítica sobre la política comercial de Washington que transmiten autores como Carmen González Martínez:

Desde mediados del siglo XIX Cuba había ido lenta pero inexorablemente pasando a ser un país dependiente de EEUU, en un proceso que había culminado en el Bill McKinley (1891). Los grandes economistas de la época señalaron 1891 como el año de la anexión de Cuba a EEUU ${ }^{86}$.

\section{CONCLUSIONES}

Después de contemplar el panorama historiográfico a ambos lados del Atlántico, más que unas conclusiones, en este apartado se ofrecen algunas sugerencias para mejorar la calidad del diálogo historiográfico a ambos lados del Atlántico.

En primer lugar, sería conveniente que los historiadores de ambos países se aventurasen más allá de sus fronteras lingüísticas. El trabajo de los historiadores norteamericanos se beneficiaría si se contextualizase mejor con la historiografía de otros países, en especial con la de un país como España, con el que los Estados Unidos mantuvieron estrechos contactos durante todo el siglo XIX. Por su parte, si los histo-

\footnotetext{
84 En Estados Unidos, un bill es un proyecto de ley.

85 ECKes, 1995, pp. 70-74.

86 González Martínez, 1998, p. 27.
} 
riadores españoles conociesen mejor la historiografía norteamericana, podrían ofrecer un retrato menos desenfocado de la política exterior de los Estados Unidos.

Un conocimiento más amplio de otras historiografías también podría ayudar a superar algunas de las dicotomías en las que sigue atrapada la historiografía norteamericana sobre la política exterior de su país. Asimismo, el peso de la influencia que según muchos historiadores recientes ejerció Gran Bretaña en la política exterior norteamericana podría ponerse en relación con el de otros países europeos.

En el caso de la historiografía española, una inmersión más profunda en la historiografía norteamericana le ayudaría a dar el salto de calidad que todavía sigue necesitando. El grado de internacionalización de la historiografía española sigue siendo todavía muy precario. Como consecuencia, ni las innovaciones historiográficas de otros países llegan con la suficiente rapidez a España, ni la producción historiográfica española sigue siendo muy conocida más allá de sus fronteras. Para superar esas barreras, es necesario empezar a participar en los debates internacionales, y no sólo tomar nota de ellos para continuar manteniendo discusiones que no tienen interés fuera de España.

El primer paso para superar este persistente nacionalismo metodológico es conocer con más precisión lo que se escribe en países como los Estados Unidos. Eso contribuiría a superar el ángulo español con el que se enfocan todas las cuestiones historiográficas y, previsiblemente, aumentar el impacto internacional de la historiografía española.

\section{REFERENCIAS BIBLIOGRÁFICAS}

BÉCKER, Jerónimo

1924-1926 Historia de las relaciones exteriores de España durante el siglo XIX. (Apuntes para una historia diplomática). Madrid. Jerónimo Ratés.

BECKER, William $\mathrm{H}$.

1973 “American Manufacturers and Foreign Markets, 1870-1900: Business Historians and the 'New Economic Determinists"'. Business History Review. Boston, no 47/4, pp. 466-481. En http://dx.doi.org/10.2307/3113367

BEISNER, Robert L.

1986 From the Old Diplomacy to the New, 1865-1900 [1975]. Arlington Heights. Harlan Davidson.

Buzzanco, Robert

1999 "What Happened to the New Left? Toward a Radical Reading of American Foreign Relations". Diplomatic History. Boulder, no 23/4, pp. 575-607. En http://dx.doi. org/10.1111/0145-2096.00186

CASTElló, Elena

2009 Juan Valera: diplomático. Madrid. Universidad Complutense de Madrid. (Tesis Doctoral inédita).

Chang, Gordon H.

2003 “'Whose 'Barbarism'? Whose 'Treachery'? Race and Civilization in the Unknown United States-Korea War of 1871". The Journal of American History. Bloomington, no 89/4, pp. 1.331-1.365. En http://dx.doi.org/10.2307/3092545 
Chapin, James B.

1971 Hamilton Fish and American Expansion. Ithaca. Cornell University. (Tesis Doctoral inédita).

CoATES, Benjamin A.

2014 "The Pan-American Lobbyist: William Eleroy Curtis and U.S. Empire, 1884-1899". Diplomatic History. Boulder, no 38/1, pp. 22-48. En http://dx.doi.org/10.1093/dh/ dht067

Companys, Julián

1991 España en 1898: entre la diplomacia y la guerra. Madrid. Ministerio de Asuntos Exteriores.

Crapol, Edward P.

2000a "Coming to Terms with Empire: The Historiography of Late Nineteenth-Century American Foreign Relations". En Hogan, Paths to Power: The Historiography of American Foreign Relations to 1941. New York. Cambridge University Press, pp. 79-116.

2000b James G. Blaine: Architect of Empire. Wilmington. Scholarly Resources.

DAVIES, Robert B.

1976 Peacefully Working to Conquer the World: Singer Sewing Machines in Foreign Markets, 1854-1920. New York. Arno Press.

DEPKAT, Volker

2003 “Cultural Approaches to International Relations: A Challenge?”. En GiENOw-Hecht - Schumacher, Culture and International History. New York. Berghahn Books, pp. 175-197.

ECKES, Alfred E.

1995 Opening America's Market: U.S. Foreign Trade Policy since 1776. Chapel Hill. University of North Carolina Press.

ElizALde, María Dolores

1992 España en el Pacífico. La colonia de las Islas Carolinas (1885-1899): un modelo colonial en el contexto internacional del imperialismo. Madrid. Consejo Superior de Investigaciones Científicas.

1997 "De nación a imperio: la expansión de los Estados Unidos por el Pacífico durante la Guerra Hispano-Norteamericana de 1898". Hispania. Madrid, nº 57/196, pp. 551588.

2000 "Balance del 98: un punto de inflexión en la modernización de España o la desdramatización de una derrota". Historia y Política. Madrid, nº 3, pp. 175-206.

2001 "E1 98 desde una perspectiva normalizadora. Reflexión historiográfica de un centenario". Hispania. Madrid, nº 61-208, pp. 707-736.

FIELD, James A.

1978 "American Imperialism: The Worst Chapter in Almost Any Book". The American Historical Review. Bloomington $\mathrm{n}^{\circ}$ 83/3, Bloomington, pp. 644-668. En http://dx. doi.org/10.1086/ahr/83.3.644

FONER, Philip S.

1972 The Spanish-Cuban-American War and the Birth of American Imperialism. New York. Monthly Review Press.

1975 La guerra hispano-cubano-americana y el nacimiento del imperialismo norteamericano, 1895-1902. Madrid. Akal. 
1978 La guerra hispano-cubano-americana y el surgimiento del imperialismo yanqui. La Habana. Editorial de Ciencias Sociales.

Fradera, Josep M.

1998 "La política colonial española del siglo XIX. (Una reflexión sobre los precedentes de la crisis de fin de siglo)". Revista de Occidente. Madrid, n 202-203, pp. 183-199.

FrISCH, Michael H.

1998 "El prisma del pasado en el cambio del siglo: uso de las ferias mundiales. La exposición panamericana de 1901 como estudio de un caso concreto". Studia Historica. Historia Contemporánea. Salamanca, nº 16, pp. 123-136.

FrY, Joseph A.

1979 "William McKinley and the Coming of the Spanish-American War: A Study of the Besmirching and Redemption of an Historical Image". Diplomatic History. Boulder, $n^{\circ} 3 / 1$, pp. 77-98. En http://dx.doi.org/10.1111/j.1467-7709.1979.tb00302.x

1996 "From Open Door to World Systems: Economic Interpretations of Late Nineteenth Century American Foreign Relations". Pacific Historical Review. Glendale, no 65/2, pp. 277-303. En http://dx.doi.org/10.2307/3639986

Gienow-Hecht, Jessica C.E.

2003 "Introduction: On the Division of Knowledge and the Community of Thought: Culture and International History". En Gienow-Hecht - Schumacher, Culture and International History. New York. Berghahn Books, pp. 3-26.

GonzÁLEZ MarTínez, Carmen

1998 "Historiografía hispano-cubana y perspectivas analíticas del 98: crisis del Estado Español”. Anales de Historia Contemporánea. Orihuela, n 14, pp. 17-31.

Gould, Lewis L.

1982 The Spanish-American War and President McKinley. Lawrence. University Press of Kansas.

1985 "Chocolate Eclair or Mandarine Manipulator? William McKinley, the Spanish-American War, and the Philippines: A Review Essay". Ohio History. Columbus, n 94, pp. 182-187.

GraEBNER, Norman A.

1985 Foundations of American Foreign Policy: A Realist Appraisal from Franklin to McKinley: Essays. Wilmington. Scholarly Resources.

HighaM, John

1989 History: Professional Scholarship in America. Baltimore. The Johns Hopkins University Press.

HiLton, Sylvia L.

1994a “Democracy Goes Imperial: Spanish Views of American Policy in 1898”. En AdAms - Minnen, Reflections on American Exceptionalism. Staffordshire. Keele University Press, pp. 97-128.

1994b “American Studies in Spain: Recent Trends". American Studies International. Washington, DC, $n^{\circ} 32 / 1$, pp. 41-69.

1998 'Democracy Beats the 'Disaster' Complex: Spanish Interpretations of the Colonial Crisis". OAH Magazine of History. Bloomington, n 12/3, pp. 11-17.

2007 "The Study of U.S. History in Spain". En Hilton - Minnen, Teaching and Studying U.S. History in Europe: Past, Present, and Future. Amsterdam. VU University Press, pp. 231-252. 
Hilton, Sylvia L. - ICKRingill, Steve J.S.

1996 "Cleveland and the Anglo-Venezuelan Dispute in 1895: A Prelude to McKinley's Intervention in the Spanish-Cuban War'. En FusI - Niño, Antes del 'Desastre': origenes y antecedentes de la crisis del 98. Madrid. Universidad Complutense de Madrid, pp. 337-358.

1998 “'Americana en letra y espíritu’: la Doctrina Monroe y el presidente McKinley en 1898”. Cuadernos de Historia Contemporánea. Madrid, no 20, pp. 205-219.

HogANSON, Kristin L.

1998 Fighting for American Manhood: How Gender Politics Provoked the Spanish-American and Philippine-American Wars. New Haven. Yale University Press.

Holbo, Paul S.

1970 "Economics, Emotion, and Expansion: An Emerging Foreign Policy". En Morgan, The Gilded Age. Syracuse. Syracuse University Press, pp. 199-221.

Hunt, Michael H.

1987 Ideology and U.S. Foreign Policy. New Haven. Yale University Press.

INAREJOS, Juan Antonio

2010 Intervenciones coloniales y nacionalismo español: la política exterior de la Unión Liberal y sus vínculos con la Francia de Napoleón III (1856-1868). Madrid. Sílex.

IRIYE, Akira

1979 "Culture and Power: International Relations as Intercultural Relations", Diplomatic History. Boulder, no 3/2, pp. 115-128. En http://dx.doi.org/10.1111/j.1467-7709.1979. tb00305.x

KaGAN, Robert

2006 Dangerous Nation. New York. Knopf.

LAFEBER, Walter

1963 The New Empire: An Interpretation of American Expansion, 1860-1898. Ithaca. Cornell University Press.

1993 The Cambridge History of American Foreign Relations: Volume 2, The American Search for Opportunity, 1865-1913. New York. Cambridge University Press.

LANGHORNE, Richard

1999 "El imperialismo: acontecimientos y procesos". Studia Historica. Historia Contemporánea. Salamanca, $\mathrm{n}^{\circ} 17, \mathrm{pp} .39-52$.

LAURIE, Bruce

1998 “'La banqueta de la penitencia': hacia una historia social del voto en el Massachusetts del siglo XIX”. Studia Historica. Historia Contemporánea. Salamanca, n 16 , pp. 67-88.

Lilley, Charles R. - Hunt, Michael H.

1987 "On Social History, the State, and Foreign Relations: Commentary on 'The Cosmpolitan Connection"”. Diplomatic History. Boulder, n 11/3, pp. 243-250. En http:// dx.doi.org/10.1111/j.1467-7709.1987.tb00016.x

MAIER, Charles S.

1980 "Marking Time: The Historiography of International Relations". En KAMmen, The Past Before Us: Contemporary Historical Writing in the United States, Ithaca, Cornell University Press, pp. 355-387. 
MARKs, Sally

1987 "The World According to Washington". Diplomatic History. Boulder, no 11/3, pp. 265-282. En http://dx.doi.org/10.1111/j.1467-7709.1987.tb00018.x

McCormick, Thomas J.

1982 "Drift or Mastery? A Corporatist Synthesis for American Diplomatic History". Reviews in American History. Baltimore, $\mathrm{n}^{\circ}$ 10/4, pp. 318-330. En http://dx.doi. $\operatorname{org} / 10.2307 / 2701835$

NinKOVICH, Frank

1982 "Ideology, the Open Door, and Foreign Policy". Diplomatic History. Boulder, n 6/2, pp. 185-208. En http://dx.doi.org/10.1111/j.1467-7709.1982.tb00371.x

1998 "No Post-Mortems for Post-Modernism, Please". Diplomatic History. Boulder, ${ }^{\circ}$ 22/3, pp. 451-466. En http://dx.doi.org/10.1111/1467-7709.00129

2010 “The New Empire”. En Kagan, The Imperial Moment. Cambridge. Harvard University Press, pp. 141-168.

2011 “Author's Response”. En Maddux - LABrosse, H-Diplo/ISSF Roundtable Review of Frank Ninkovich. Global Dawn: The Cultural Foundation of American Internationalism, 1865-1890. 3/3. En http://www.h-net.org/ diplo/ISSF/PDF/ISSF-Roundtable-3-3.pdf

Novick, Peter

1988 That Noble Dream: The "Objectivity Question” and the American Historical Profession. Cambridge. Cambridge University Press.

OFFNER, John L.

1992 An Unwanted War: The Diplomacy of the United States and Spain over Cuba, 18951898. Chapel Hill. University of North Carolina Press.

1997 "La política norteamericana y la guerra hispano-cubana”. En FUSI - NiÑo, Vísperas del 98: orígenes y antecedentes de la crisis del 98. Madrid. Biblioteca Nueva, pp. 195-203.

PALEN, Marc-William

2010 "Protection, Federation and Union: The Global Impact of the McKinley Tariff Upon the British Empire, 1890-94". Journal of Imperial \& Commonwealth History. London, $n^{\circ} 38 / 3$, pp. 395-418. En 10.1080/03086534.2010.503395

2013 “Foreign Relations in the Gilded Age: A British Free-Trade Conspiracy?". Diplomatic History. Boulder, no 37/2, pp. 217-247. En http://dx.doi.org/10.1093/dh/dht003

2015 “The Imperialism of Economic Nationalism, 1890-1912”. Diplomatic History. Boulder, no 39/1, pp. 157-185. En http://dx.doi.org/10.1093/dh/dht135

PANDO, Juan

1996 "Las conversaciones Prim-Sickles: España-Cuba-Estados Unidos en 1868-70". En FusI - NiÑo, Antes del 'Desastre'. Orígenes y antecedentes de la crisis del 98. Madrid. Universidad Complutense de Madrid, pp. 359-377.

PARDO, Rosa

2003 "La política norteamericana". Ayer. Madrid, no 49, pp. 13-53.

PATERSON, Thomas G.

1996 "United States Intervention in Cuba, 1898: Interpretations of the Spanish-American-Cuban-Filipino War". The History Teacher. Long Beach, n 29/3, pp. 341-361. En 10.2307/494551

PÉrez, Louis A., Jr.

1983a Cuba between Empires: 1878-1902. Pittsburgh. University of Pittsburgh Press. 
1983b "Toward Dependency and Revolution: The Political Economy of Cuba between Wars, 1878-1895". Latin American Research Review. Alburquerque, no 18/1, pp. 127-142.

1989 "The Meaning of the Maine: Causation and the Historiography of the Spanish-American War”. Pacific Historical Review. Glendale, n 58/3, pp. 293-322. En http://dx. doi.org/ 10.2307/3640268.

PIQUeRAs, José Antonio

2003 Cuba, emporio y colonia: la disputa de un mercado interferido (1878-1895). Madrid. Fondo de Cultura Económica.

Pletcher, David M.

1962 The Awkward Years: American Foreign Relations under Garfield and Arthur. Columbia. University of Missouri Press.

1978 "Reciprocity and Latin American in the 1890s: A Foretaste of Dollar Diplomacy". Pacific Historical Review. Glendale, $\mathrm{n}^{\circ}$ 47-1, pp. 53-89. En http://dx.doi. org $/ 10.2307 / 3637339$

1981 "Rethoric and Results: A Pragmatic View of American Economic Expansionism, 1865-1898". Diplomatic History. Boulder, no 5/2, pp. 93-106. En http://dx.doi. org/10.1111/j.1467-7709.1981.tb00773.x

1984 "1861-1898: Economic Growth and Diplomatic Adjustment”. En Becker - Wells, Economics and World Power: An Assesment of American Diplomacy since 1789. New York. Columbia University Press, pp. 119-171.

1990 “Caribbean 'Empire': Planned and Improvised”. Diplomatic History. Boulder, no 14/3, pp. 447-460. En http://dx.doi.org/10.1111/j.1467-7709.1990.tb00101.x

PRIEST, Andrew

2014 "Thinking about Empire: The Administration of Ulysses S. Grant, Spanish Colonialism and the Ten Years' War in Cuba". Journal of American Studies. Cambridge, $\mathrm{n}^{\mathrm{o}}$ 48/2, pp. 541-558. En http://dx.doi.org/10.1017/S0021875813002004

RESTAD, Hilde Eliassen

2012 "Old Paradigms in History Die Hard in Political Science: US Foreign Policy and American Exceptionalism". American Political Thought. Chicago, n 1/1, pp. 53-76. En http://dx.doi.org/10.1086/664586

Robles, Cristóbal

1990 "La lucha de los independentistas cubanos y las relaciones de España con Estados Unidos". Hispania. Madrid, no 50/174, pp. 159-202.

1991 1898: diplomacia y opinión. Madrid. Consejo Superior de Investigaciones Científicas.

RoLDÁN, Inés

2000 La Restauración en Cuba: el fracaso de un proceso reformista. Madrid. Consejo Superior de Investigaciones Científicas.

2003 "La política española en Cuba: una década de cambios (1876-1886)". Ayer. Madrid, $\mathrm{n}^{\mathrm{o}} 52$, pp. 175-203.

ROSENBERG, Emily S.

1982 Spreading the American Dream: American Economic and Cultural Expansion, 18901945. New York. Hill and Wang. 
RuBIO, Javier

1995 La cuestión de Cuba y las relaciones con los Estados Unidos durante el reinado de Alfonso XII: los orígenes del "desastre" de 1898. Madrid. Ministerio de Asuntos Exteriores.

2004 El final de la era de Cánovas: los preliminares del "desastre” de 1898. Madrid. Ministerio de Asuntos Exteriores.

SALOM, Julio

1967 España en la Europa de Bismarck. La política exterior de Cánovas, 1871-1881. Madrid. Consejo Superior de Investigaciones Científicas.

SÁNCHEZ ANDRÉs, Agustín

1998 "Crisis colonial y política exterior española en la cuenca del Caribe (1878-1898)". Revista Mexicana del Caribe. Chetumal, nº 5, pp. 6-30.

2007 "Una diplomacia defensiva: la política exterior española en el Caribe y el Golfo de México entre 1865 y 1878". Hispania. Madrid, nº 67/226, pp. 487-516. En http:// dx.doi.org/10.3989/hispania.2007.v67.i226.51

SANTAMaría, Antonio - NARAnjo, Consuelo

1999 "El '98 en América. Últimos resultados y tendencias recientes de la investigación”. Revista de Indias. Madrid, no 59-215, pp. 203-274. En http://dx.doi.org/10.3989/revindias. 1999.i215.745

SCHLESINGER, Arthur M., Jr.

1986 The Cycles of American History. Boston. Houghton Mifflin.

SCHMIDT-NowARA, Christopher

1998 “Imperio y crisis colonial”. En PAn-Montojo, Más se perdió en Cuba. España, 1898 y la crisis de fin de siglo. Madrid. Alianza Editorial, pp. 31-90.

1999 "El mito liberal del Imperio: España, Cuba y el 98”. Studia Historica. Historia Contemporánea. Salamanca, nº 17, pp. 53-63.

SCHOONOVER, Thomas D.

1991 The United States in Central America, 1860-1911: Episodes of Social Imperialism and Imperial Rivalry in the World System. Durham. Duke University Press.

SCHWARTz, Thomas A.

2007 "Explaining the Cultural Turn -or Detour". Diplomatic History. Boulder, no 31/1, pp. 143-147. En http://dx.doi.org/10.1111/j.1467-7709.2007.00594.x

SEXTON, Jay

2003 "The Funded Loan and the Alabama Claims". Diplomatic History. Boulder, n 27/4, pp. 449-478. En http://dx.doi.org/10.1111/1467-7709.00364

2006 "The United States, the Cuban Rebellion, and the Multilateral Initiative of 1875 ". Diplomatic History. Boulder, $\mathrm{n}^{\circ}$ 30/3, pp. 335-365. En http://dx.doi.org/10.1111/ j.1467-7709.2006.00559.x

SHUBERT, Adrian

2004 "Spanish Historians and English-speaking Scholarship". Social History. London, $\mathrm{n}^{\circ}$ 29/3, pp. 358-363. En http://dx.doi.org/10.1080/0307102042000257638

STEPHANSON, Anders

1998 "Commentary: Diplomatic History in the Expanded Field". Diplomatic History. Boulder, no 22/4, pp. 595-603. En http://dx.doi.org/10.1111/0145-2096.00140

TERrILl, Tom E.

1973 The Tariff, Politics, and American Foreign Policy, 1874-1901. Westport. Greenwood Press. 
THELEN, David

1999 "La experiencia vivida, un reto para la historia profesional moderna: californianos del sur e historiadores científicos ante el cambio de siglo". Studia Historica. Historia Contemporánea. Salamanca, $\mathrm{n}^{\circ} 17$, pp. 145-172.

Thompson, J.A.

1973 "William Appleman Williams and the 'American Empire'". Journal of American Studies. Cambridge, $\mathrm{n}^{\mathrm{o}}$ 7/1, pp. 91-104. En http://dx.doi.org/10.1017/ S0021875800012640.

UCELAY-Da CAL, Enric

1994 "The Hispanic Studies 'Ghetto"”. Bulletin of the Society for Spanish and Portuguese Historical Studies. San Diego, nº 19/3, pp. 11-15.

VV.AA.

1998 La crisis del 98. Madrid. Centro de Información y Documentación Científica.

WiLKIns, Mira

1970 The Emergence of Multinational Enterprise: American Business Abroad from the Colonial Era to 1914. Cambridge. Harvard University Press.

WiLliams, William A.

1959 The Tragedy of American Diplomacy. Cleveland. The World Publishing Co.

ZAKARIA, Fareed

1998 From Wealth to Power: The Unusual Origins of America's World Role. Princeton. Princeton University Press. 